\title{
Millimeter-Wave Textile-Based Monopole Antenna for Wearable Wireless Power Transmission
}

\author{
Mahmoud Wagih, Alex S. Weddell, and Steve Beeby \\ School of Electronics and Computer Science, University of Southampton, Southampton, SO17 1BJ, U.K. \\ https://www.ecs.soton.ac.uk/
}

\begin{abstract}
With a wide bandwidth and a high potential for antenna miniaturization, the millimeter-wave (mmWave) bands have attracted interest for a range of wearable applications. In this paper, a textile-based end-fire monopole is proposed for wireless power transmission (WPT) applications. The proposed Yagi-inspired antenna bandwidth covers the 24 $\mathrm{GHz}$ license-free band. The antenna achieves $73 \%$ total efficiency, a $2.5 \mathrm{~dB}$ improvement over a microstrip patch on the same textile substrate. The antenna has a peak gain over $4 \mathrm{dBi}$ with a wide $80^{\circ}$ half-power beamwidth both in space and on a layered human tissue model. The antenna is experimentally characterized for line-of-sight WPT showing a $7 \mathrm{~dB}$ forward transmission improvement compared to $2.45 \mathrm{GHz}$ WPT, for $55 \mathrm{~cm}$ separation, between two symmetric antennas with sub- $\mathrm{cm}^{2}$ area. Given the antenna's $0.4 \mathrm{~cm}^{2}$ area, it is shown that mmWaves enable high-efficiency WPT to miniaturized antennas in future $5 \mathrm{G}$ networks.
\end{abstract}

\section{Introduction}

Millimeter-wave (mmWave) bands are increasingly recognized as an enabler of future wireless networks [1]. Body area networks (BANs) operating in the mmWave bands have been investigated for high-speed on and off-body links [2]. Moreover, mmWave energy harvesting has attracted significant interest where high gain transmitting and receiving antennas can improve the end-to-end efficiency in wireless power transmission (WPT) compared to UHF bands [3].

mmWave rectennas and antennas have been widely developed for WPT and energy harvesting in the K/Ka-bands using a variety of implementations. For instance, a cavitybacked circularly polarized array [4], a broadband textilebased antipodal vivaldi antenna [5], flexible and printed microstrip patch arrays [6, 7], and a Rotman lens-fed microstrip array [8] were proposed for mmWave WPT. For mmWave antennas operating on lossy substrates, such as 3D printable flexible packages and textiles, achieving a high radiation efficiency is a significant challenge due to the high dielectric $(\tan \delta)$ losses.
Textile-based mmWave antennas have been reported with varying radiation efficiencies, typically under $70 \%$ due to the lossy textile substrates [9, 10, 11, 5]. End-fire and broadside textile-based antennas have been proposed for 60 $\mathrm{GHz}$ on- [10] and off-body [9] communications, respectively, achieving under 50\% radiation efficiency. In [5], a reflector-backed antenna was proposed for energy harvesting, achieving around $67 \%$ radiation efficiency owing to its thicker substrate and lack of ground-plane backing. Recently, an experimental characterization of on-body UHF and mmWave links have shown that the excess delay can be reduced by a factor of three at $60 \mathrm{GHz}$ compared to 900 $\mathrm{MHz}$, with comparable path-loss when using $60 \mathrm{GHz}$ horn antennas [12].

In this paper, a Yagi-inspired microstrip-fed monopole antenna is proposed for WPT and energy harvesting applications in future $5 \mathrm{G}$ bands, achieving a wide bandwidth and a high radiation efficiency. The antenna maintains a wide $S_{11}<-10 \mathrm{~dB}$ bandwidth from 22 to $26 \mathrm{GHz}$ covering the license-free $24 \mathrm{GHz}$ band with a $73 \%$ total antenna efficiency, demonstrating a higher efficiency compared to reported textile-based mmWave antennas. The antenna design and simulated parameters are presented in Section 2, Section 3 then presents the experimental validation, as well as WPT measurements between symmetric antennas in a Line-of-Sight (LoS) link.

\section{Antenna Design and Simulation}

The antenna is designed for the $24 \mathrm{GHz} \mathrm{K}$-band Industrial Scientific and Medical (ISM) band, previously considered for various rectenna implementations $[4,6]$. The antenna is designed based on a $\lambda / 4$ monopole with an unconnected reflector and 9 director elements, fabricated on a single metal layer. The number of directors was selected as a tradeoff between achieving a medium peak gain, a wide beamwidth, and for keeping the antenna compact. Additional directors can be added to increase the antenna's on-body (end-fire) gain.

Figure 1 shows the layout and dimensions of the proposed antenna. The antenna is designed for a $300 \mu \mathrm{m}$-thick polyimide-on-woven polyester textile-based substrate. The substrate's dielectric properties were measured using two 

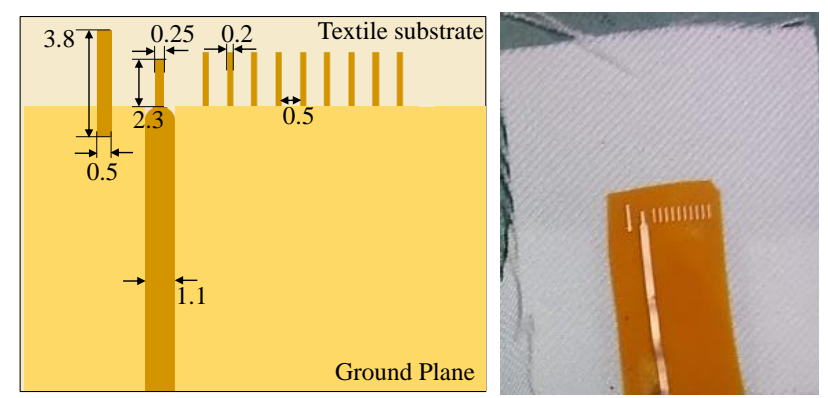

Figure 1. Layout and dimensions (in $\mathrm{mm}$ ) of the antenna and photograph of the fabricated prototype on textile.

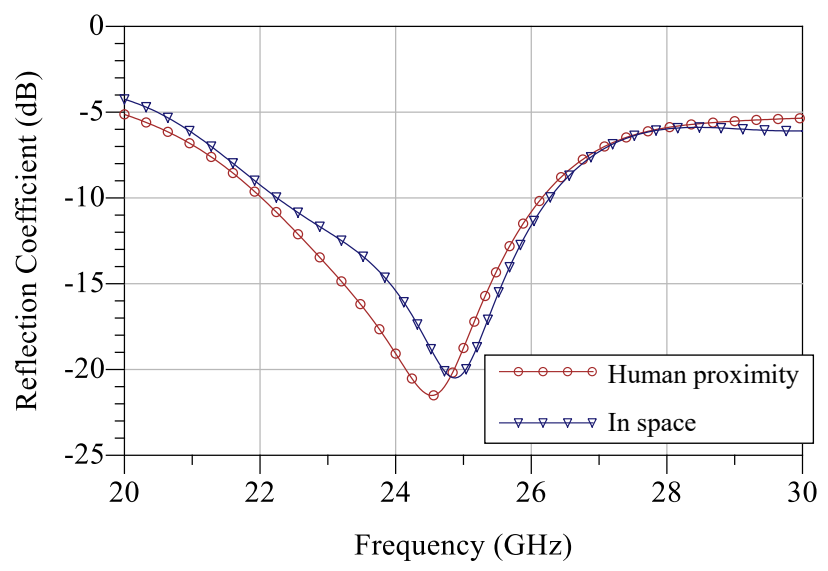

Figure 2. Simulated reflection coefficient $\left(S_{11}\right)$ of the proposed antenna.

microstrip lines on the same substrate to be $\varepsilon_{r}=1.95$ and $\tan \delta=0.02$, in line with most woven-textile substrates previously used for wearable antennas $[9,5]$. A prolonged microstrip feed was added to match the fabricated prototype, where the prolonged feed reduces the unwanted reflections off the coaxial connector $[9,5]$, which is significantly larger than the antenna's radiator. The antenna occupies a total area of approximately $10 \times 4 \mathrm{~mm}$, excluding the prolonged microstrip feed.

The antenna was simulated in CST Microwave Studio. As the antenna is intended for wearable applications, a layered tissue model similar to [5], based on the previously reported experimental mmWave dielectric properties of skin [13], was included to observe the human-proximity effects on the antenna's $S_{11}$ and radiation properties. The tissue model was placed at $4 \mathrm{~mm}$ from the antenna. Figure 2 shows the simulated $S_{11}$ of the antenna in the presence and absence of the tissue model, demonstrating a stable and wide bandwidth from 22 to $26 \mathrm{GHz}$, covering the $24 \mathrm{GHz}$ ISM-band. The minimal variation in the simulated $S_{11}$ in human proximity is attributed to the antenna's wide bandwidth and the reflection of the mmWave radiation off-skin.

The radiation patterns of the antenna were simulated in CST, with and without the tissue model. Figure 3 shows the simulated realized gain (inclusive of impedance mismatch)

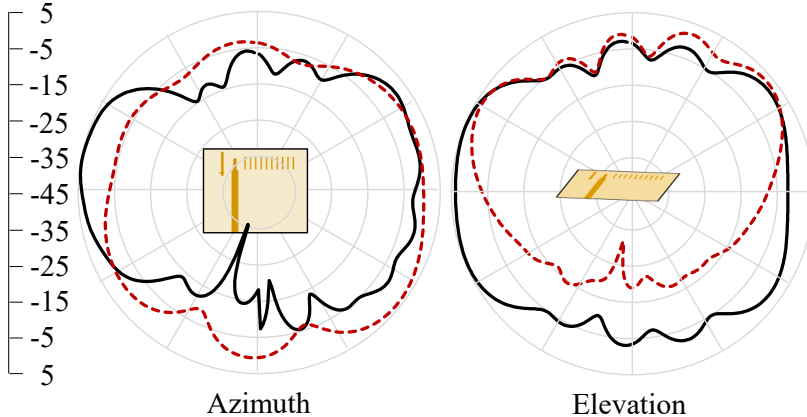

Figure 3. Simulated realized gain patterns (in $\mathrm{dBi}$ ) of the proposed antenna in space (solid) and in human-proximity (dashed).

over the azimuth and elevation planes. The peak realized gain is 4.8 and $4.6 \mathrm{dBi}$ in absence and presence of the tissue model, respectively.

From the gain patterns, it can be observed that the antenna maintains a wide beam-width over both planes, with over $80^{\circ} 3 \mathrm{~dB}$ beam-width on the azimuth plane. A wide beam was previously found to improve the energy harvesting efficiency from unknown directions [14], and is more generally considered a figure-of-merit in wearable communications [15]. The peak end-fire gain is validated through the WPT measurement in the next section.

A $\mathrm{TM}_{10}$ common geometry microstrip patch antenna was simulated on the same textile substrate, to enable evaluation the improvement in the radiation efficiency, achieved by the proposed design. The patch's dimensions are $L=4.3$ and $W=4 \mathrm{~mm}$. Both antennas were fed using a short 3 $\mathrm{mm}$ microstrip line to minimize any feed-induced insertion losses, enabling a better evaluation of the antenna's own losses [9]. At $24 \mathrm{GHz}$, the proposed antenna achieves a $73 \%$ efficiency with the $3 \mathrm{~mm}$ microstrip feed. On the other hand, the microstrip patch achieves a $40 \%$ efficiency, owing to the increased dielectric losses in the $\tan \delta=0.02$ substrate. Therefore, the proposed antenna represents around $2.5 \mathrm{~dB}$ efficiency improvement over a $\mathrm{TM}_{10}$ patch, explaining the higher efficiency over previously-reported textilebased mmWave antennas such as $[5,9,10]$.

\section{Antenna Measurements}

The antenna was fabricated using photolithography on a commercially-available flexible polyimide copper laminate using the method described in [5]. The antenna's traces were then adhered to the woven-polyester textile substrate using a spray-mount adhesive. A low-temperature solder was then used to mount the connector in order to avoid damaging the textile substrate.

The antenna was characterized using an Agilent E8361A $67 \mathrm{GHz}$ PNA Vector Network Analyser (VNA) with a standard SOLT electronic calibration. A solder-terminated connector was used to connect the antenna to the VNA. As 


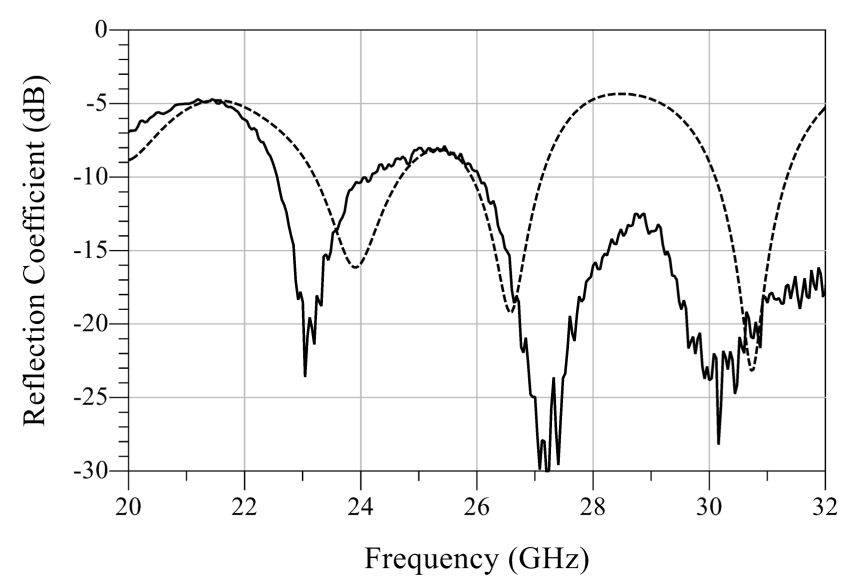

Figure 4. Simulated (dashed) and measured (solid) reflection coefficient of the antenna with a solder-terminated edge connector

the connector's unshielded pin is of comparable size to $\lambda / 4$ around $26 \mathrm{GHz}$, which may alter the impedance $\left(S_{11}\right)$ response, the antenna needs to be simulated with the connector [5]. Figure 4 shows the simulated and measured reflection coefficient of the antenna with the mounted connector. Both the simulated and measured demonstrate the antenna maintains an $S_{11}<-10 \mathrm{~dB}$ at $24 \mathrm{GHz}$. Furthermore, when considering the VSWR $<3\left(S_{11}<-6 \mathrm{~dB}\right)$ bandwidth, it can be observed that the antenna's bandwidth spans the full $5 \mathrm{G}$ $\mathrm{K} / \mathrm{Ka}$-band spectrum up to $32 \mathrm{GHz}$.

The next step in characterizing the antenna is investigating its performance in a LoS point-to-point WPT link, demonstrating that a higher WPT efficiency can be achieved at mmWave bands. The power received by an antenna from an incident Poynting vector (RF power density) $S$ is given by

$$
P_{\mathrm{RX}}=S A_{\text {eff. }},
$$

where $A_{\text {eff }}$ is the antenna's effective area. Given that $A_{\text {eff }}$ is limited by the aperture efficiency of an aperture antenna (e.g. horn or microstrip patch) [16], (1) can be rewritten as

$$
P_{\mathrm{RX}}=A_{\mathrm{TX}} A_{\mathrm{RX}} \eta_{\mathrm{TX}} \eta_{\mathrm{RX}} \frac{f^{2}}{c^{2} d^{2}}
$$

where $f$ is the frequency, $c$ is the speed of light, and $\eta$ and $A$ are the antennas' aperture efficiency and physical area respectively [3].

From (2), it can be observed that for compact transmitters and receivers of a fixed physical area $A$, operating at a higher $f$ enables a better forward transmission between the antennas and subsequently a higher end-to-end efficiency in WPT. Two symmetric antennas based on the proposed monopole were connected to a VNA's ports at a fixed distance $d=55 \mathrm{~cm}$. The $S_{21}$ was measured from 1 to 40 GHz. Figure 5 shows the measured $S_{21}$ over the antenna's impedance bandwidth.

At $23.5 \mathrm{GHz}$, where the maximum $S_{21}=-47 \mathrm{~dB}$ was measured, and for $d=55 \mathrm{~cm}$, the free space path-loss is $54.7 \mathrm{~dB}$.

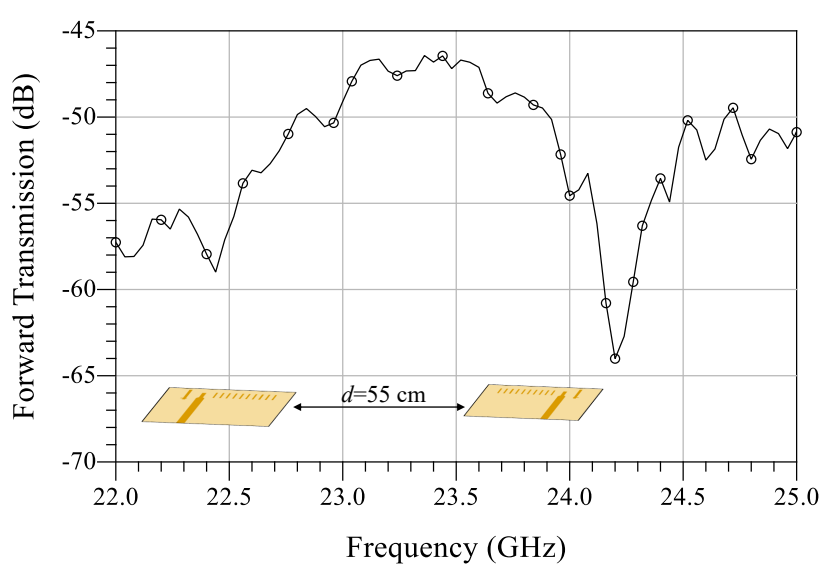

Figure 5. Measured forward transmission between two symmetric antennas at $d=55 \mathrm{~cm}$.

Hence the measured gain of the antenna, using the twoantennas method [16], is $3.9 \mathrm{dBi}$, agreeing with the peak simulated gain presented in Section 3.

The mmWave $S_{21}$ between the compact antennas is compared to that at UHF bands. At $2.45 \mathrm{GHz}$, and after excluding the impedance mismatch losses, a forward transmission of $-55 \mathrm{~dB}$ was measured. Given the $35 \mathrm{~dB}$ path-loss at $2.45 \mathrm{GHz}$, the antennas' gain is around $-10 \mathrm{dBi}$ in the 2.4 GHz ISM-band, showing that for a wireless power transmitter and receiver restricted to sub- $1 \mathrm{~cm}^{2}$ area, a significantly higher gain and subsequently $P_{\mathrm{RX}}$ can be achieved by operating in the $24 \mathrm{GHz}$ band. From a WPT perspective, while the RF to DC efficiency is lower at mmWave bands compared to UHF [3], several recent rectifier implementations have shown efficiencies over $60 \%$ up to $35 \mathrm{GHz}$ [17].

\section{Conclusion}

In this paper, a textile-based microstrip antenna was presented for wearable WPT. The proposed antenna achieves an $S_{11}$ bandwidth covering the $24 \mathrm{GHz}$ ISM-band and over $4 \mathrm{dBi}$ gain with a broad beam in both the presence and absence of human tissue. The antenna's performance for UHF and mmWave WPT has been compared showing over $7 \mathrm{~dB}$ forward transmission improvement, for the same separation and antenna physical area, by operating in the $24 \mathrm{GHz}$ band. Finally, the antenna's high radiation efficiency of up to $73 \%$ and its small form-factor demonstrate that the proposed antenna is suitable for mmWave WPT applications in future $5 \mathrm{G}+$ networks.

\section{Acknowledgements}

This work was supported by the UK Engineering and Physical Sciences Research Council (EPSRC) under Grant EP/P010164/1. 


\section{References}

[1] I. A. Hemadeh, K. Satyanarayana, M. El-Hajjar, and L. Hanzo, "Millimeter-Wave Communications: Physical Channel Models, Design Considerations, Antenna Constructions, and Link-Budget," IEEE Comms Surv. Tutorials, vol. 20, 2, pp. 870 - 913, 2017.

[2] N. Chahat, M. Zhadobov, L. L. Coq, S. I. Alekseev, and R. Sauleau, "Characterization of the Interactions Between a 60-GHz Antenna and the Human Body in an Off-Body Scenario," IEEE Trans. Antennas Propag., vol. 60 no. 12, pp. 5958 - 5965, 2012.

[3] M. Wagih, A. S. Weddell, and S. Beeby, "MillimeterWave Power Harvesting: A Review," IEEE Open Journal of Antennas and Propagation, vol. 1, pp. 560 $-578,2020$.

[4] S. Ladan, A. B. Guntupalli, and K. Wu, "A HighEfficiency $24 \mathrm{GHz}$ Rectenna Development Towards Millimeter-Wave Energy Harvesting and Wireless Power Transmission," IEEE Trans. Circuits And Systems, vol. 61, 12, pp. 3358 - 3366, 2014.

[5] M. Wagih, G. S. Hilton, A. S. Weddell, and S. Beeby, "Broadband Millimetre-Wave Textile-based Flexible Rectenna for Wearable Energy Harvesting ," IEEE Trans. Microw Theory Techn, vol. 68 no. 11, pp. 4960 - 4972, 2020.

[6] J. Bito, V. Palazzi, J. Hester, R. Bahr, F. Alimenti, P. Mezzanotte, L. Roselli, and M. M. Tentzeris, "Millimeter-wave ink-jet printed RF energy harvester for next generation flexible electronics," in 2017 IEEE Wireless Power Transfer Conference (WPTC), 2017.

[7] T.-H. Lin, S. N. Daskalakis, A. Georgiadis, and M. M. Tentzeris, "Achieving fully autonomous system-onpackage designs: An embedded-on-package 5g energy harvester within $3 \mathrm{~d}$ printed multilayer flexible packaging structures," in 2019 IEEE MTT-S International Microwave Symposium (IMS), 2019.

[8] A. Eid, J. Hester, and M. M. Tentzeris, "A Scalable High-Gain and Large-Beamwidth mm-Wave Harvesting Approach for 5G-powered IoT," in 2019 IEEE MTT-S International Microwave Symposium (IMS), 2019.

[9] N. Chahat, M. Zhadobov, S. A. Muhammad, L. L. Coq, and R. Sauleau, "60-GHz Textile Antenna Array for Body-Centric Communications," IEEE Trans. Antennas Propag., vol. 61 no. 4, pp. 1816 - 1824, 2013.

[10] N. Chahat, M. Zhadobov, L. L. Coq, and R. Sauleau, "Wearable Endfire Textile Antenna for On-Body Communications at $60 \mathrm{GHz}$," IEEE Antennas Wireless Propag. Lett., vol. 11, pp. 799 - 802, 2012.

[11] M. Ur-Rehman, N. A. Malik, X. Yang, Q. H. Abbasi, Z. Zhang, and N. Zhao, "A Low Profile Antenna for
Millimeter-Wave Body-Centric Applications," IEEE Trans. Antennas Propag., vol. 65 no. 12, pp. $6329-$ 6337, 2017.

[12] R. Aminzadeh, A. Thielens, M. Zhadobov, L. Martens, and W. Joseph, "WBAN Channel Modeling for $900 \mathrm{MHz}$ and $60 \mathrm{GHz}$ Communications," IEEE Trans. Antennas Propag., 2020 Early access, DOI: 10.1109/TAP.2020.3045498.

[13] D. Ghodgaonkar, O. Gandhi, and M. Iskander, "Complex permittivity of human skin in vivo in the frequency band 26.5-60 GHz," in IEEE Antennas and Propagation Society International Symposium, 2000.

[14] M. Wagih, O. Cetinkaya, B. Zaghari, A. S. Weddell, and S. Beeby, "Real-World Performance of Sub$1 \mathrm{GHz}$ and $2.4 \mathrm{GHz}$ Textile Antennas for RF-Powered Body Area Networks," IEEE Access, vol. 8, pp. 133746 - $133756,2020$.

[15] M. Joler and M. Boljkovac, "A Sleeve-Badge Circularly Polarized Textile Antenna," IEEE Trans. Antennas Propag., vol. 66 no. 3, pp. 1576 - 1579, 2018.

[16] Yi Huang and Kevin Boyle, Antennas: From Theory to Practice. West Sussex, United Kingdom: John Wiley \& Sons Ltd, 2008.

[17] H. Mei, X. Yang, B. Han, and G. Tan, "Highefficiency microstrip rectenna for microwave power transmission at Ka band with low cost," IET Microwave Antennas and Propagation, vol. 10 no. 15, pp. 1648-1655, 2016. 